\title{
Excluded Volume Approximation For Supernova Matter
}

\author{
A.V. Yudin* \\ Institute for Theoretical and Experimental Physics, Moscow, Russia \\ E-mail: yudineitep.ru
}

\begin{abstract}
Starting from a simple modification of ordinary Fermi-gas description we develop a general approach to the excluded volume approximation (EVA). This approach takes into account the arbitrary degree of particle degeneracy and permits the inclusion of other interactions. We introduce the effective excluded volume function, whose form can be chosen in different ways to obtain various models. In particular it is easy to obtain a well-known EVA which was used in the heavy ion reactions study. By implementing our general approach to the case of Boltzmann limit we can reproduce the results of well-designed theory of hard-sphere particles and describe the behavior of many-component different sized mixtures. By adding to the EVA an additional long-ranged attractive potential we have obtained quasi Van der Waals equation of state and use it to explore thermodynamic properties and chemical composition of matter in collapsed supernova cores. The ability of above approach to describe a phase transition to uniform nuclear matter is also considered.
\end{abstract}

11th Symposium on Nuclei in the Cosmos, NIC XI

July 19-23, 2010

Heidelberg, Germany

\footnotetext{
* Speaker.
} 


\section{Introduction}

Even at high densities up to the nuclear density value $\rho \sim 10^{14} \mathrm{~g} / \mathrm{cm}^{3}$, the chemical composition of matter is the mixture of different nuclei. Thus one needs to account for theirs finite size (the existence of strong repulsive nuclear interaction at short distance). The real nucleon potential can be approximated by a hard-core repulsive part and long-ranged attractive term. This means that the excluded volume approximation (EVA) is the most simple approach to the description of the matter into a subnuclear domain. Every model of EVA must satisfy the following conditions: first, it must allow the description of different-sized particles mixture. Second, it must reproduce the action of nuclear forces in sub-nuclear domain and ensures the smooth transition from low-density region of weak interaction to high-density strong interacting nuclear matter. And the last: it must permit the inclusion of other interactions, for example the Coulomb one.

\section{General approach}

Let us assume that the particles are fermions of $M$ sorts in volume $V$. The number of states for the particle on $n$-th sort in phase-space is $V G_{n}^{k}, G_{n}^{k}=g_{n} d^{3} p_{k} / h^{3}$, where $g_{n}=\left(2 j_{n}+1\right)$ and index $k$ numbers the cells in the momentum space. If in this cell there are $N_{n}^{k}$ particles then the statistical weight is equal [1]:

$$
\ln \triangle \Gamma_{n}^{k}=G_{n}^{k} V \ln G_{n}^{k} V-\left[G_{n}^{k} V-N_{n}^{k}\right] \ln \left(G_{n}^{k} V-N_{n}^{k}\right)-N_{n}^{k} \ln N_{n}^{k} .
$$

We modify this equation in the spirit of excluded volume approximation. Namely we change $V \rightarrow V\left(1-v_{n}\right)$ where $v_{n}=v_{n}\left(n_{1}, n_{2}, . ., n_{M}\right)$ and $n_{i}$ is the concentration of $i$-th sort of particles. After this we define $\triangle \Gamma_{n}=\prod_{k} \triangle \Gamma_{n}^{k}$ and find the entropy according to $S=\sum_{n} S_{n}, S_{n}=k \ln \triangle \Gamma_{n}$. Next we apply the variational principle i.e. find the entropy maximum under the conditions of fixed particle numbers $N_{n}=\sum_{k} N_{n}^{k}$ and total energy $E=\sum_{n, k} \varepsilon_{n}^{k} N_{n}^{k}$ :

$$
\delta\left(S+\sum_{n} \alpha_{n} N_{n}+\beta E\right)=0, \quad \alpha_{n}=\frac{\mu_{n}}{k T}, \quad \beta=-\frac{1}{k T} .
$$

Here $\alpha_{n}$ and $\beta$ are the Lagrange multipliers and $\mu_{n}$ are the chemical potentials. Performing variation procedure we find:

$$
N_{n}^{k}=\frac{G_{n}^{k} V\left(1-v_{n}\right)}{1+\exp \left[\left(\varepsilon_{n}^{k}-\widetilde{\mu}_{n}\right) / k T\right]}, \quad \widetilde{\mu}_{n}=\mu_{n}-\sum_{p}\left(\frac{\partial v_{p}}{\partial n_{n}}\right) P_{p}^{\mathrm{id}}\left(T, \widetilde{\mu}_{p}\right) .
$$

thus we obtain the Fermi-distribution in reduced volume $V^{*}=V\left(1-v_{n}\right)$ as a function of modified chempotential $\widetilde{\mu}_{n}$, where $P_{p}^{\mathrm{id}}\left(T, \widetilde{\mu}_{p}\right)$ stands for the ideal Fermi-gas pressure expression as a function of $T$ and $\mu$. By the summation over $\mathrm{k}$ in the first equation (2.3) we find $n_{n}=\left(1-v_{n}\right) n^{\mathrm{id}}\left(T, \widetilde{\mu}_{n}\right)$, which together with the second equation in (2.3) implicitly determines the composition. It must be stressed that now equilibrium equations form the non-linear system. After solving this equations we can find all the thermodynamic quantities: energy, pressure, etc., for example:

$$
E=V \sum_{n}\left(1-v_{n}\right) E_{n}^{\mathrm{id}}\left(T, \widetilde{\mu}_{n}\right), \quad P=\sum_{n}\left[1-v_{n}+\sum_{p}\left(\frac{\partial v_{n}}{\partial n_{p}}\right) n_{p}\right] P_{n}^{\mathrm{id}}\left(T, \widetilde{\mu}_{n}\right) .
$$


Equilibrium equations (2.3) together with the thermodynamic ones (2.4) completely determine the state of the system. It can be proven that the system of equations (2.3-2.4) ensures the thermodynamical consistency (i.e fulfil the identities $n=\left(\frac{\partial P}{\partial \mu}\right)_{T}$ etc.) for any functional form $v_{n}=v_{n}\left(n_{1}, n_{2}, . ., n_{M}\right)$.

Various extra interactions can be taken into account by the free energy approach $F^{\text {tot }}=F_{0}+$ $\triangle F$, where $F_{0}$ - free energy of a system without interaction and $\triangle F$ is the addition caused by interaction. Then other thermodynamic quantities will get corresponding additions, for example $\triangle S=-\left(\frac{\partial \triangle F}{\partial T}\right)_{N, V}$, etc.

How does it work? A little example: let us assume that excluded volumes are the same for all particle kinds and equal simply to the occupied volume: $v_{n}=\sum_{k} v_{k} n_{k}$ where $v_{k}$ is the particle's own volume. Then we obtain:

$$
P=\sum_{n} P_{n}^{\mathrm{id}}\left(T, \widetilde{\mu}_{n}\right)=P^{\mathrm{id}}\left(T,\left\{\widetilde{\mu}_{n}\right\}\right), \quad \widetilde{\mu}_{n}=\mu_{n}-v_{n} P^{\mathrm{id}}\left(T,\left\{\widetilde{\mu}_{n}\right\}\right),
$$

This is the well-known EVA-description of [2].

The results of this section can be summarize as follows: using the formulas $(2.3-2.4)$ one can choose some specific form of excluded volume function $v_{n}\left(n_{1}, n_{2}, . ., n_{M}\right)$, add extra interaction through free energy formalism described above and obtain his own model of EVA.

\section{Hard-Sphere Liquids}

For the Boltzmann particles interacting through the hard-core potential there exist a number of theoretical and numerical results and we've tried to connect our approach in this limit to the model of hard-sphere liquids.

\subsection{One-component case}

In the one-component case the only parameter characterized EVA is packing number $\eta$. In the general case $\eta=\frac{\pi}{6} \sum_{n} \sigma_{n}^{3} n_{n}$ where $\sigma$ is the particle diameter, thus $\eta$ is just the ratio of the volume, occupied by the particles to the whole one. The main quantity characterized the systems of hard spheres is $\Gamma$ :

$$
\Gamma \equiv \frac{P}{k T \sum_{i} n_{i}}, \quad \Gamma_{\mathrm{oc}}(\eta)=\frac{1+\eta+\eta^{2}-\eta^{3}}{(1-\eta)^{3}}
$$

The last expression for the one-component case $\Gamma_{\mathrm{oc}}$ was derived in [3]. Then using equation (2.4) for the pressure in Boltzmann limit we can find:

$$
v(\eta)=1-\exp \left[-\int_{0}^{\eta} \frac{\Gamma(x)-1}{x} d x\right]
$$

Thus in the Boltzmann limit our approach can reproduce the results of hard-sphere liquids theory. 


\subsection{Multi-component case}

To obtain the results for multi-component case we postulate the demands for EVA: when the diameter of some component tends to zero, it must be described by the formulas for an ideal gas with $\widetilde{\mu}_{n}=\mu_{n}$ but in the smaller volume $V^{*}=V(1-\eta)$. Next: in the low-density limit we must be able to reproduce the first terms of virial expansion which can be obtained analytically. And last: if the diameters of all components are equal, we must come to the one-component case (3.2).

Now we introduce a new set of functions $\psi_{n}$ according to the identity $1-v_{n} \equiv \exp \left(-\psi_{n}\right)$. Because in the low-density limit $v_{n} \approx \psi_{n}$ we will try this form for $\psi_{n}$ :

$$
\psi_{n}=\frac{\pi}{6} \sum_{i} n_{i} \widehat{\sigma}_{n i}^{3} G\left(\eta, z_{n i}\right), G=\frac{g_{1}(\eta)+z_{n i} g_{2}(\eta)}{\eta}, z_{n i}=\frac{2 \sigma_{n} \sigma_{i}}{\sigma_{n}+\sigma_{i}} \frac{\left\langle\sigma^{2}\right\rangle}{\left\langle\sigma^{3}\right\rangle}, \widehat{\sigma}_{n i}^{3} \equiv \sigma_{i}\left(\sigma_{i}+\sigma_{n}\right)^{2}
$$

Here we introduce the average values according to $\left\langle\sigma^{k}\right\rangle \equiv \sum_{i} \sigma^{k} n_{i} / \sum_{i} n_{i}$. After some mathematical manipulations we can find:

$$
\begin{gathered}
\psi_{n}=\left[1+2 \sigma_{n} \frac{\left\langle\sigma^{2}\right\rangle}{\left\langle\sigma^{3}\right\rangle}+\sigma_{n}^{2} \frac{\langle\sigma\rangle}{\left\langle\sigma^{3}\right\rangle}\right] g_{1}(\eta)+2 \sigma_{n} \frac{\left\langle\sigma^{2}\right\rangle}{\left\langle\sigma^{3}\right\rangle}\left[1+\sigma_{n} \frac{\left\langle\sigma^{2}\right\rangle}{\left\langle\sigma^{3}\right\rangle}\right] g_{2}(\eta) . \\
g_{1}(\eta)=-\ln (1-\eta), \quad g_{2}(\eta)=\int_{0}^{\eta}\left[\Gamma_{\mathrm{oc}}(x)-\frac{1+3 x}{1-x}\right] \frac{d x}{4 x},
\end{gathered}
$$

Now we can use this expressions and find the value of $\Gamma$ parameter for the multi-component system in Boltzmann limit:

$$
\Gamma=\frac{1}{1-\eta}+\frac{\langle\sigma\rangle\left\langle\sigma^{2}\right\rangle}{2\left\langle\sigma^{3}\right\rangle}\left\{\left[\Gamma_{o c}(\eta)-\frac{1}{1-\eta}\right]\left(1+\frac{\left\langle\sigma^{2}\right\rangle^{2}}{\langle\sigma\rangle\left\langle\sigma^{3}\right\rangle}\right)+\frac{3 \eta}{1-\eta}\left(1-\frac{\left\langle\sigma^{2}\right\rangle^{2}}{\langle\sigma\rangle\left\langle\sigma^{3}\right\rangle}\right)\right\} .
$$

This expression for $\Gamma$ exactly coincides with the one obtained in [4].

\section{The applications}

We apply the formulas of the previous section to the case of supernova matter under the conditions of NSE in the subnuclear densities domain. For the diameters of particles we accept the following values: $\sigma_{n}=\sigma_{p}=1.6 \mathrm{fm}$ for free neutrons and protons and $\sigma_{A, Z}=2.32 A^{1 / 3} \mathrm{fm}$ for nuclei (here $A$ is the nucleus mass number). On the subsequent graphs (except the phase diagram plot) temperature is always $T=5 \mathrm{MeV}$ and $Y_{\mathrm{e}}=\frac{1}{3}$.

Figure 1 shows the chemical composition of an ideal matter (solid lines) and a matter with the excluded volume interaction according to the hard-sphere model (dashed) as a function of density $\rho .{ }^{89} \mathrm{Se}$ is the most neutron-rich nucleus in our set of nuclides. On the figure 2 we plot the calculated value of packing number $\eta$. It is clear that excluded volume effect itself has only a minor influence on the equilibrium concentrations even in the domain where the value of $\eta$ is comparable with 1 .

Figure 3 shows the behavior of pressure's relative deviation $\left(P-P^{\mathrm{id}}\right) / P^{\mathrm{id}}$ where $P^{\mathrm{id}}$ stands for the pressure of ideal matter. Red line, marked EV corresponds to the excluded volume model. By the blue line (marked EV+LRA) is shown the model with excluded volume correction and an 


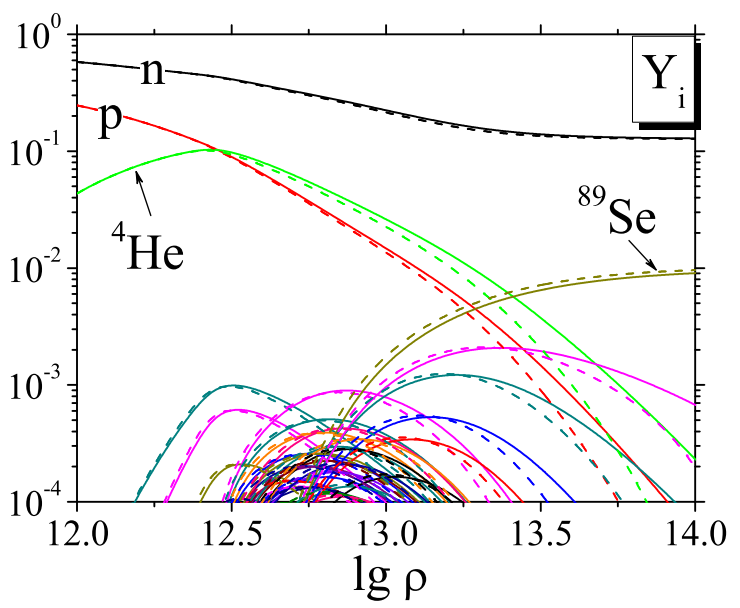

Figure 1: Equilibrium concentrations

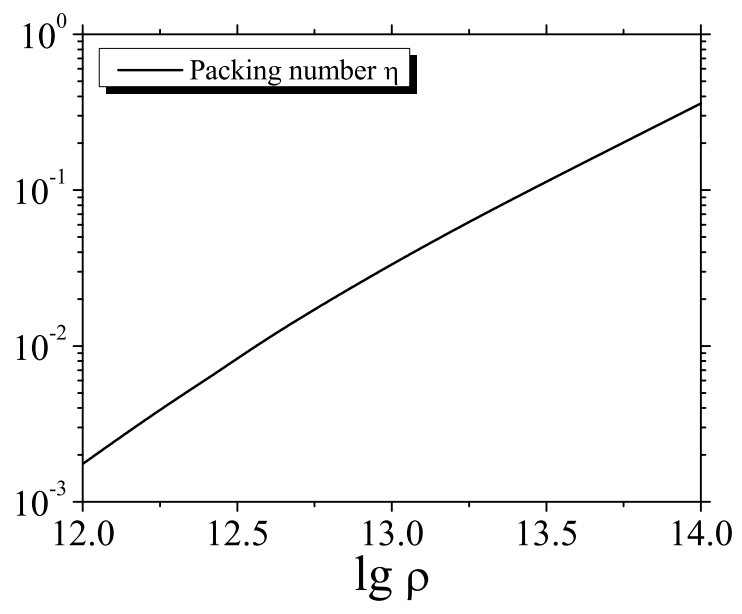

Figure 2: Packing number $\eta$

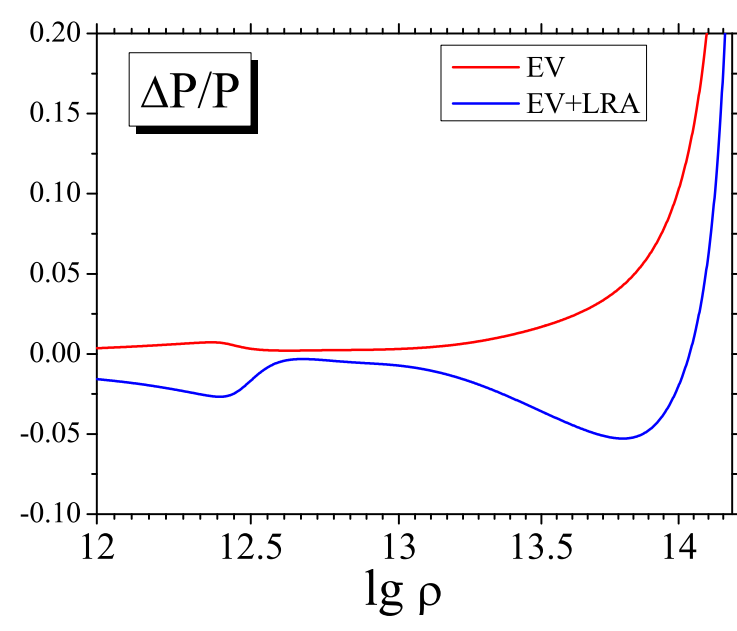

Figure 3: Relative pressure deviation

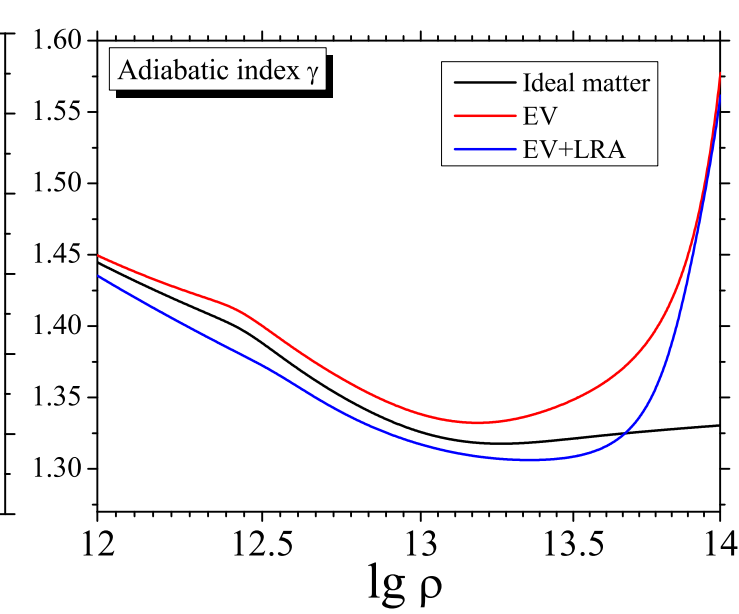

Figure 4: Adiabatic index $\gamma$

additional long-ranged Yukawa-type attractive potential which was introduced to reproduce the correct form of nucleon-nucleon interaction. Non-monotonicity of $\triangle P$ with the density in EV model is caused by the equilibrium concentrations changes.

Figure 4 represents the adiabatic index $\gamma$ of matter. For an ideal matter the value of $\gamma$ is shown by black line. Excluded volume effect always tends to stabilize the matter as it should, but EV+LRA model predicts slightly lower values of $\gamma$ in low-density domain. This is caused by attractive part of interaction potential. At densities $\rho \sim 10^{14}$ both models predict the rapid growth of $\gamma$ and the matter become stiff. This is the domain where the phase transition to uniform nuclear matter must take place. At higher densities an excluded volume models become inapplicable.

\subsection{Phase transition}

In this section we show how our approach can be applied to the problem of phase transition. Of course, to obtain the quantitative results one should use a well-adjusted EOS'es in both phases. The further calculations are only the example. For the low-density domain we use EV+LRA model, 
described above, and for the high-density region we apply the expression for uniform nuclear matter from [5]. Figure 5 shows the phase diagram of the system for the values of $Y_{\mathrm{e}}: \frac{1}{2}$ - black lines,

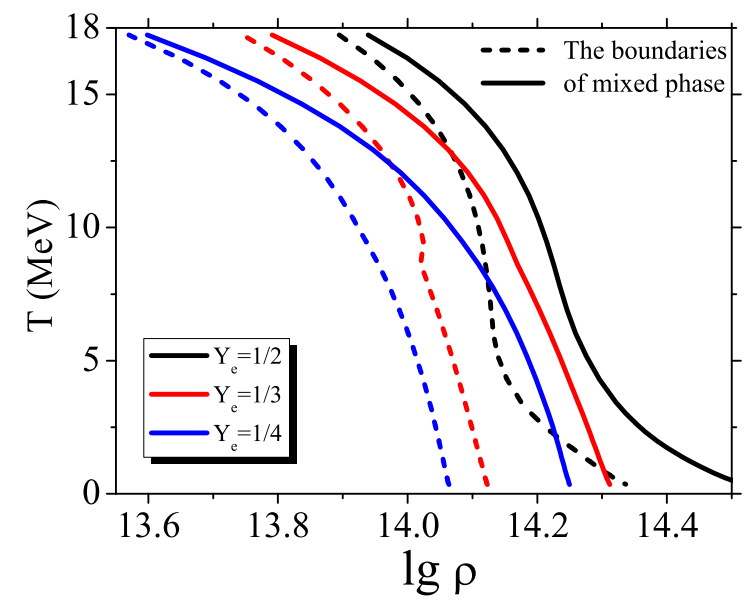

Figure 5: Phase diagram

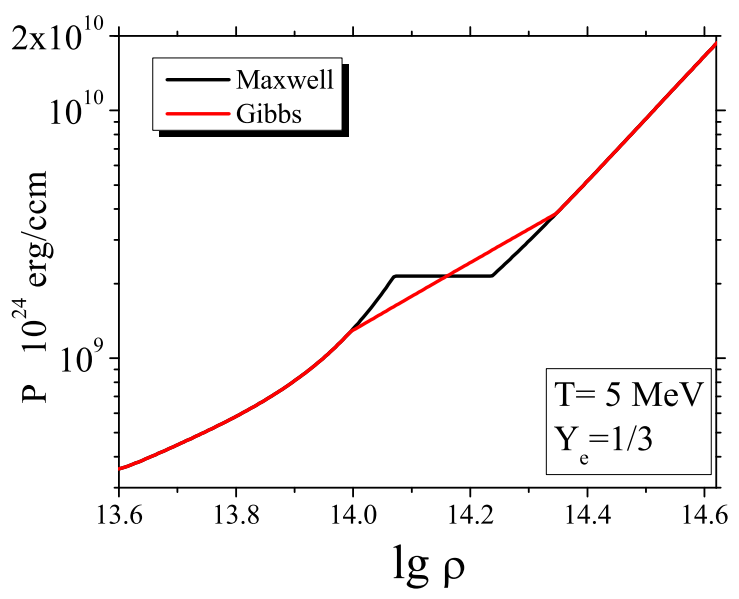

Figure 6: Pressure at phase transition

$\frac{1}{3}$ with red lines and $\frac{1}{4}$ - blue. Dashed lines correspond to the boundary between the low-density phase and the mixed phase region, solid lines - to the boundary between mixed phase and uniform nuclear matter. This phase diagram was obtained within the Maxwell approach to the description of phase transition.

On the figure 6 is shown the pressure-density dependence calculated using ordinary Maxwell's construction (black line) and quasi-Gibbs approach (red line). For the Gibbs approach pressure in the mixed phase is not constant but the mixed phase domain is wider.

\section{Conclusions}

The approach to the excluded volume approximation developed here can serve as a useful tool for exploring the properties of matter under the extreme conditions. By varying the form of excluded volume function and choosing the appropriate additional interaction one can easily obtain various thermodynamically consistent EOS models.

Using this EVA approach it occurs possible to reproduce (in the Boltzmann limit) the results of well-designed theory of hard-sphere liquids. This model seems to be an adequate approximation for the multi-component mixture of free nucleons and nuclei under the conditions of NSE - the case of supernova matter in the sub-nuclear domain. Thus, one can not only to investigate the thermodynamic properties of matter but also to derive the detailed information about its chemical composition. This is a big advantage of EVA compared to the common "average nucleus" models. The nucleosynthesis problems can be solved only via this kind of EOS'es.

Besides this, we have demonstrated that it is also possible to explore the properties of phase transition phenomenon using this EVA. Our own supernova core-collapse calculations utilizing the EVA equation of state described here are underway. 


\section{Acknowledgments}

I am grateful to the NIC-XI Organizing Committee for the possibility to attend the Conference. I would also thank M. Liebendörfer for his hospitality during my stay in Basel and visit to Heidelberg. The work was supported by the grants: RFASI No. 02.740.11.0250 and SNSF SCOPES project No IZ73Z0-128180/1.

\section{References}

[1] L.D. Landau, E.M. Lifshitz, Statistical Physics Vol. 1, Moscow 1976.

[2] D.H. Rischke, M.I. Gorenstein, H. Stocker, W. Greiner, Excluded volume effect for the nuclear matter equation of state, Z. Phys. C 51, 4851991.

[3] N.F. Carnahan, K.E. Starling, Equation of State for Nonattracting Rigid Spheres , J. Chem.Phys. 51, 6351969.

[4] M. Lopez de Haro, S.B. Yuste and A. Santos, Theory and Simulation of Hard-Sphere Fluids and Related Systems, Lecture Notes in Physics, Vol 753, p 183. Springer-Verrlag Berlin Heidelberg, 2008.

[5] J.M. Lattimer, F.D. Swesty, A generalized equation of state for hot, dense matter, Nucl. Phys. A 535, 331-376, 1991. 